\title{
Feedback Effect of Exertion Value on Accuracy of Knee Extension Strength Exertion Values
}

\author{
Hiroki Aoki ${ }^{*}$, Shin-Ichi Demura² \\ ${ }^{1}$ National Institute of Technology, Fukui College, Fukui, Japan \\ ${ }^{2}$ Graduate School of Natural Science \& Technology, Kanazawa University, Kanazawa, Japan \\ Email: *aoki@fukui-nct.ac.jp
}

How to cite this paper: Aoki, H., \& Demura, S.-I. (2017). Feedback Effect of Exertion Value on Accuracy of Knee Extension Strength Exertion Values. Advances in Physical Education, 7, 487-493. https://doi.org/10.4236/ape.2017.74041

Received: February 8, 2017

Accepted: November 26, 2017

Published: November 29, 2017

Copyright $\odot 2017$ by authors and Scientific Research Publishing Inc. This work is licensed under the Creative Commons Attribution International License (CC BY 4.0).

http://creativecommons.org/licenses/by/4.0/

\section{(c) (i) Open Access}

\begin{abstract}
Feedback of strength exertion value may increase the accuracy of the next strength exertion value. This study examined the effect of exertion value feedback on the accuracy of knee extension strength exertion values. The subjects were 14 healthy young males (mean age $21.0 \pm 6.1$ years, mean height $171.9 \pm 8.6 \mathrm{~cm}$, mean mass $66.1 \pm 3.4 \mathrm{~kg})$. Three demand values $(25 \%, 50 \%$, and $75 \%$ ) of maximum voluntary contraction (MVC) were selected. First, after we measured the MVC of knee extension strength in the dominant leg, subjects performed ten trials for each demand value without exertion value feedback. Then, they performed ten trials and received exertion value feedback on each trial for each demand value. Differences (errors) between the demand and exerted values for each trial, a mean error for the first and second trials (the first mean) and the ninth and tenth trials (the last mean), and a change ratio (the first mean/the last mean) were calculated. Regression coefficients among errors in each trial and ten trials were significant for all demand values only in the feedback condition. In addition, the interception was significant for all demand values, but not between conditions with/without feedback. The last mean error was significantly less with than without feedback for all demand values, and it was less than the first mean error in the 50\% MVC. In addition, the change rate was significantly less with feedback only in the $25 \%$ MVC. In conclusion, when exertion value feedback is provided, the accuracy of knee extension strength exertion values is high. In addition, accuracy increases with trials but is marked in the lowest (25\%) MVC.
\end{abstract}

\section{Keywords}

Isokinetic Strength, Feedback, Accuracy

\section{Introduction}

In daily life, people exert submaximal strength while coordinating (Kubota \& 
Demura, 2012), and the degree of strength exertion is generally determined by a subjective, experiential “sense”. Existing studies on exertion values' accuracy when subjects adjustably exert submaximal strength while matching demand values have been mainly performed for static strength (Ohashi, 1994; Kitabayashi et al., 2013). In short, the effect of feedback on the accuracy of dynamic strength exertion has not been comprehensively studied. After examining differences between demand and exertion values in isokinetic knee extension strength using dominant and non-dominant legs, Demura et al. (2001) reported no significant difference between demand values of $25 \%, 50 \%$, and $75 \%$ of maximum strength in both legs. However, with visual feedback on exertion values, the next exertion value's accuracy increased.

When persons estimate a degree of strength exertion, their motor order registers in the kinesthetic memory, and both feed-forward regulation and feedback inhibition relate to muscle output response (Brooks, 1986). Presumably, when subjects receive strength exertion value feedback, they can easily memorize exertion degree, thereby increasing accuracy compared with when no feedback is received. Ohashi (1994) also reported that when subjects received exertion value feedback, their accuracy of grip strength exertion increased with repeated trials. Presumably, when there are no effects of fatigue and boredom, feedback on results generally enhances exertion value accuracy with repeated trials. Ohashi's results may also apply to the strength exertion of lower limbs. According to Seki and Ohtsuki's (1995) report, an error between demand value and strength exertion value based on subjective judgment tends to become higher as the demand value increases.

However, presumably, when feedback is provided, exertion value accuracy is high for strength exertion approaching maximum strength because persons use not only somatosensory information but also feedback information.

This study examined the effect of exertion value feedback on the accuracy of knee extension strength exertion value.

\section{Methods}

1) Subjects

The subjects included 14 young male athletes (mean age $21.0 \pm 6.1$ years, mean height $171.9 \pm 8.6 \mathrm{~cm}$, mean mass $66.1 \pm 3.4 \mathrm{~kg}$ ) with competitive experience of over four years in swimming, soccer, and so on. All subjects were judged as having a stronger right leg by Sato and Demura's (2011) dominant leg survey. We explained the purpose, methods, and risks of the experiment to the subjects and obtained their consent. The Ethics Committee on Human Experimentation of Faculty of Human Science, Kanazawa University approved the protocol for this study (Approval number: 2012-02).

2) Experimental Equipment and Methods

Knee extension strength was measured using a Cybex-325 (Simizu Medical, Japan). This device suspends an attachment to each joint's range of motion, 
measuring the subject's isokinetic strength.

Data measured by the Cybex-325 was relayed to a personal computer through a cable and analyzed with exclusive software (Simizu Medical, HUMAC 2009). The measurements were performed on the subjects' dominant legs, using a velocity of $60 \mathrm{deg} / \mathrm{sec}$. We measured knee extension strength twice and used a greater value as a parameter. In consideration of an order effect, after randomly dividing subjects into six groups, each group from the first to the sixth was measured according to six patterns: 1) $25 \% \rightarrow 50 \% \rightarrow 75 \%$ MVC; 2) $25 \% \rightarrow 75 \% \rightarrow$ $50 \%$ MVC; 3) $50 \% \rightarrow 25 \% \rightarrow 75 \%$ MVC; 4) $50 \% \rightarrow 75 \% \rightarrow 25 \%$ MVC; 5) $75 \% \rightarrow$ $25 \% \rightarrow 50 \%$ MVC; and 6) $75 \% \rightarrow 50 \% \rightarrow 25 \%$ MVC.

Subjects performed ten trials for each demand value. For example, for the first group (pattern (1)), after performing ten trials with 25\% MVC, they did the same with 50\% MVC, and then with 75\% MVC. In addition, we calculated a relative value [(exertion value-demand value)/maximal value] and provided subjects the value after each trial to create a feedback condition.

3) Parameters

Presumably, when subjects repeat ten trials for each demand value, errors between demand and exertion values decrease as the trials progress, but they are even fewer with feedback.

We calculated the following: differences (errors) between the demand and exerted values for each trial, a mean error of the first and second trials (the first mean) and the ninth and tenth trials (the last mean), and a change ratio (the first mean/the last mean). In this study, the first and last mean errors and the change rate were used as parameters.

4) Data Analysis

A linear regression equation was calculated to examine the relationship between trials and each trial error; then, the significance of the coefficient was tested. A three-way repeated measures ANOVA (feedback on or off $\times$ the first and last two trials $\times$ demand values [25\%, 50\%, and 75\% MVC]) was used to examine differences between the first and last mean errors. A two-way repeated measures ANOVA (feedback on or off $\times$ the first and last two trials) was used to examine differences between mean errors to determine change rate. When a significant interaction or main effect was found, a Tukey's honestly significant difference (HSD) test was used for a multiple comparison, and the level of significance was determined to be 0.05 .

\section{Results}

Figure 1 shows the plots each error (absolute value) with/without feedback for each demand value along with their regression equations. Regression coefficients among errors in each trial and ten trials were significant for all demand values only in the feedback condition. In addition, the interception showed significance for all demand values but non-significant difference between conditions with/without feedback. 
Table 1 displays the basic statistics of mean errors with/without feedback, the first and last means, and the results of the two-way ANOVA. A significant interaction was found, and a multiple comparison showed that the last mean error was less with than without feedback for all demand values as well as less than the first mean error in 50\% MVC.

Table 2 displays the basic statistics of change rates with/without feedback, demand values, and the results of the two-way ANOVA. A significant main effect (with/without feedback) was found only for feedback, and it was less without feedback only in the $25 \%$ MVC.
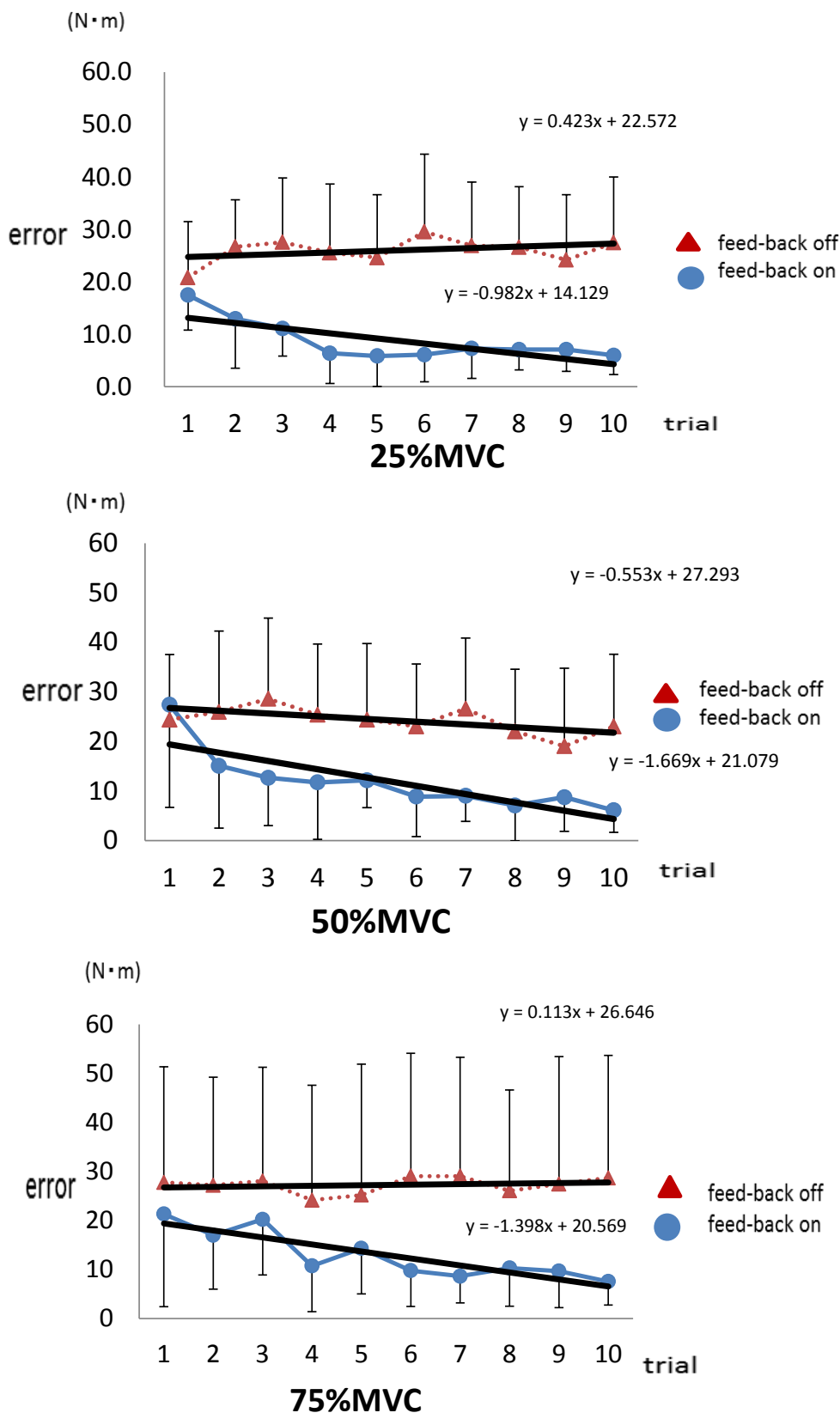

Figure 1. Plot of each error (absolute value) with/without feedback for each demand value and their regression equations. 
Table 1. Basic statistics of mean errors with/without feedback, first and last means, and results of two-way ANOVA.

\begin{tabular}{|c|c|c|c|c|c|c|c|c|}
\hline & & \multicolumn{2}{|c|}{ first trial } & \multicolumn{2}{|c|}{ last trial } & \multicolumn{2}{|c|}{ F-value } & \multirow{3}{*}{$\begin{array}{c}\text { Post-hoc } \\
25 \%, 50 \%, 75 \% \text { MVC } \\
\text { (last trial): on }<\text { off } \\
\text { on } 50 \% \text { MVC: first } \\
\text { trial }>\text { last trial }\end{array}$} \\
\hline & & Mean & SD & Mean & SD & $\mathrm{F} 1$ & $17.65^{\star}$ & \\
\hline $25 \%$ & $\begin{array}{l}\text { feed-back off } \\
\qquad(\mathrm{N} \cdot \mathrm{m})\end{array}$ & 23.8 & 7.5 & 25.8 & 12.1 & $\mathrm{~F} 2$ & 0.15 & \\
\hline MVC & $\begin{array}{l}\text { feed-back on } \\
\qquad(\mathrm{N} \cdot \mathrm{m})\end{array}$ & 15.2 & 5.6 & 6.5 & 3.2 & F3 & 1.95 & \\
\hline $50 \%$ & $\begin{array}{l}\text { feed-back off } \\
\qquad(\mathrm{N} \cdot \mathrm{m})\end{array}$ & 25.2 & 13.4 & 21 & 14.5 & $\mathrm{~F} 4$ & 0.85 & \\
\hline MVC & $\begin{array}{l}\text { feed-back on } \\
\qquad(\mathrm{N} \cdot \mathrm{m})\end{array}$ & 21.3 & 14.4 & 7.4 & 3.7 & F5 & 0.39 & \\
\hline $75 \%$ & $\begin{array}{l}\text { feed-back off } \\
\qquad(\mathrm{N} \cdot \mathrm{m})\end{array}$ & 27.5 & 21.2 & 28.1 & 25 & F6 & $7.4^{*}$ & \\
\hline MVC & $\begin{array}{l}\text { feed-back on } \\
\qquad(\mathrm{N} \cdot \mathrm{m})\end{array}$ & 19.2 & 13.7 & 8.6 & 4.3 & F7 & $6.77^{\star}$ & \\
\hline
\end{tabular}

${ }^{\star} p<0.05$; Unit: N.m; F1: with/without feedback; F2: the first and last two trials; F3: demand values; F4: interaction (with/without feedback $\times$ the first and last two trials); F5: interaction (with/without feedback $\times$ demand values); F6: interaction (the first and last two trials $\times$ demand values); F7: interaction (with/without feedback $\times$ the first and last two trials $\times$ demand values).

Table 2. Basic statistics of change rate with/without feedback, demand values, and results of two-way ANOVA.

\begin{tabular}{|c|c|c|c|c|c|c|c|}
\hline & \multicolumn{2}{|c|}{ Feed-back off } & \multicolumn{2}{|c|}{ Feed-back on } & \multirow{2}{*}{\multicolumn{2}{|c|}{ F-value }} & \multirow{2}{*}{ post-hoc } \\
\hline & Mean & SD & Mean & $\mathrm{SD}$ & & & \\
\hline $\begin{array}{c}25 \% \text { MVC change } \\
\text { ratio }(\%)\end{array}$ & 115.5 & 60.3 & 48.2 & 29.2 & $\mathrm{~F} 1$ & $29.59^{\star}$ & $25 \%$ off $>$ on \\
\hline $\begin{array}{c}50 \% \text { MVC change } \\
\text { ratio (\%) }\end{array}$ & 93.7 & 80.6 & 39.9 & 19.9 & F2 & 0.66 & \\
\hline $\begin{array}{c}75 \% \text { MVC change } \\
\text { ratio }(\%)\end{array}$ & 100 & 46.1 & 63.9 & 39.1 & F3 & 0.67 & \\
\hline
\end{tabular}

${ }^{*} p<0.05 ;$ Unit: \%; F1: with/without feedback; F2: demand values; F3: interaction.

\section{Discussion}

Bilodeau (1966) reported that feedback can be provided to subjects as external knowledge of results. Thus, in this study, we orally informed the subjects of their exerted values and examined the feedback's effect on accuracy of knee extension isokinetic strength exertion. "Grading ability" subjectively controls physical output strength and assigns gradations to this strength. In this study, we used different demand values (25\%, 50\%, and 75\% MVC) and examined whether the effect of exertion value feedback (ten trials) on dynamic strength exertion value differs between demand values.

Regression coefficients among errors in each trial and ten trials and interception were significant in all demand values only in the feedback condition. How- 
ever, the interception showed non-significance between conditions with/without feedback. This result suggests that regardless of difference in demand values, the first-trial exertion value exhibits no significant difference; however, errors between demand and exertion values decrease with repeated trials when subjects are provided information on results. In short, the accuracy of exertion value is enhanced.

In addition, in any demand value, no significant difference was found between the first mean errors, but the later mean errors were fewer in the feedback on condition than in the feedback off condition. These results indicate that when subjects did not receive feedback on results, their exertion value accuracy did not change even when repeating trials, regardless of difference in demand values. In contrast, when subjects received feedback, their exertion value accuracy increased with trials. In short, the exertion values gradually approach the demand value. This result seems to depend on coordinating the next exertion value by referring to the previous exertion value. In addition, Ohashi (1994) reported that when subjects received knowledge of results in a static-grip strength-coordination task, the error between exertion and demand values decreased. As subjects can memorize the degree of their own strength exertion, it is inferred that they can revise it easily when provided feedback on results; thus, errors concerning exertion and demand values shrink as the number of trials rises.

On the other hand, when frequently provided feedback information, learners depend on it and find it difficult to revise active movement by using motion perception occurring during reaction; as a result, they cannot perform accurate movement (Schmidt et al., 1989). Although in this study, subjects performed ten trials of adjustable strength exertion, the effect was found in the last mean error only when feedback was given. If considerable feedback is provided, generally, exertion value is similar to requirement value, and the effect reaches its limit. This study used ten trials, but according to the results, an error may decrease through repeated trials.

In the present results, for demand values of $25 \%, 50 \%$, and $75 \% \mathrm{MVC}$, the change rate differed between with/without feedback only with 25\% MVC. In cases of low-demand value compared with those of high-demand value approaching maximum strength, subjects may find it difficult to estimate the amount of strength exertion. The effect becomes marked, particularly when feedback is not provided: even if subjects repeat trials, errors between the demand and exertion values may not shrink. Thus, the change rate in 25\% MVC may differ between conditions with/without feedback. On the other hand, according to Oonishi et al. (2008), subjects cannot recognize and control strength exertion as isometric strength exertion increases.

In the cases of relatively high demand values of 50\% and 75\% MVC used in this study, because subjects can skillfully control strength exertion even with exertion value feedback, and because errors between demand and exertion values do not decrease, their change rate may not have differed between feedback on 
and off conditions.

\section{Conclusion}

When feedback is provided, the accuracy of knee extension strength exertion values is high. In addition, accuracy increases with trials, but it is notable in the lowest $25 \%$ MVC.

\section{References}

Bilodeau, E. A. (1966). Acquisition of Skill. New York: Academic Press, 352-353.

Brooks, V. B. (1986). The Neural Basis of Motor Control. New York: Oxford University Press Inc., 142-148.

Demura, S., Yamaji, S., Goshi, F., \& Nagasawa, Y. (2001). Lateral Dominance of Legs in Maximal Muscle Power, Muscular Endurance, and Grading Ability. Perceptual and Motor Skills, 93, 11-23.

Kitabayashi, T., Demura, S., \& Aoki, H. (2013). Differences between Maximum and Submaximum Pinch Strengths in Dominant and Non-Dominant Hands. Gazzetta Medica Italiana Archivio per le Scienze Mediche, 172, 179-184.

Kubota, H., \& Demura, S. (2012). Laterality and Differences in Controlled Force Exertion among Competitive Sports Athletes. Gazzetta Medica Italiana Archivio per le Scienze Mediche, 171, 103-108.

Ohashi, Y. (1994). The Influence of Intertrial Interval on Learning of an Isometric Contractile Task. Japanese Journal of Psychology, 65, 215-213. https://doi.org/10.4992/jjpsy.65.215

Oonishi, K., Hase, K., \& Obinata, G. (2008). Analysis of Human Control Ability for Isometric Force. Transactions of the JSME, 74, 163-170.

Sato, S., \& Demura, S. (2011). Characteristics of the Subjective, Stabilization and Mobilization Preference Leg: The Relationship between These Preference Legs and the Leg Used for Stabilization and Mobilization Activities. Gazetta Medica Italiana, 171, 123-130.

Schmidt, R. A., Young, D. E., Swinnen, S., \& Shapiro, D. C. (1989). Summary Knowledge of Results for Skill Acquisition: Support for the Guidance Hypothesis. Journal of Experimental Psychology: Learning, Memory, and Cognition, 15, 352-359. https://doi.org/10.1037/0278-7393.15.2.352

Seki, T., \& Ohtsuki, T. (1995). Reproducibility of Subjectively Graded Voluntary Isometric Muscle Strength in Unilateral and Simultaneous Bilateral Exertion, Ergonomics, 38, 1867-1876. https://doi.org/10.1080/00140139508925234 